\title{
Minimum Principles for Interacting Cosserat Elastic Continua
}

\author{
D. Ieşan ${ }^{1}$ and A. Scalia ${ }^{2}$ \\ 1 "O. Mayer" Institute of Mathematics, Romanian Academy, 700506 Iaşi, Romania \\ ${ }^{2}$ Department of Mathematics and Computer Science, University of Catania, Viale A Dori, No. 6, 95125 Catania, Italy
}

Correspondence should be addressed to D. Ieşan; iesan@uaic.ro

Received 24 November 2014; Accepted 14 February 2015

Academic Editor: Alex Elías-Zúñiga

Copyright (c) 2015 D. Ieşan and A. Scalia. This is an open access article distributed under the Creative Commons Attribution License, which permits unrestricted use, distribution, and reproduction in any medium, provided the original work is properly cited.

\begin{abstract}
This paper is concerned with the linear theory of composites modelled as mixtures of two Cosserat elastic continua. First, we present a minimum principle in the case of equilibrium. Then, we consider the dynamic theory and establish a minimum principle of Reiss type for a mixed problem.
\end{abstract}

\section{Background}

In order to describe adequately the behaviour of some kinds of mixtures it is necessary to introduce into the continuum theory terms reflecting the microstructure of the materials. In a realistic continuum model, each particle of a granular material or a solid containing microscopic components (e.g., nanocomposites) possesses six degrees of freedom. Important among such materials are animal bones, solid with microcracks, and other synthetic materials with pores or microreinforcements. The origin of the modern theories of mixtures of materials with microstructure goes back to the papers of Allen and Kline [1], Twiss and Eringen [2, 3], and Dunwoody [4]. For a review of the literature on mixtures with microstructure the reader is referred to $[5,6]$. In many papers the mechanical behaviour of composites is modelled as mixtures of interacting continua (see, e.g., [7-10] and references therein). A general theory of mixtures with microstructure has been established in [2,3]. In [3], the nonlinear constitutive equations for mixtures of micromorphic and micropolar elastic bodies are derived. The results are used to derive the linear theory of mixtures composed of two Cosserat elastic constituents. The equations presented in $[2,3,6]$ are sufficiently general to apply to any constituents, whether solid or fluid, in eulerian description. A theory of a mixture of two Cosserat elastic solids in lagrangian description has been established in [10]. This theory allows us to formulate the boundary conditions in the reference configuration.
In the present paper we consider the linear theory of mixture of two Cosserat elastic solids. In Section 2 we present the notations and the basic equations. Section 3 is devoted to a minimum principle in the equilibrium theory. In Section 4 we consider the dynamic theory and establish a minimum principle of Reiss type. Variational characterizations of solutions in nonpolar theories of mixtures have been presented in various papers (see, e.g., $[5,11-15]$ and references therein).

\section{Methods Mathematical Formulation}

We consider a body which is made up of two interpenetrating elastic solids of Cosserat type $s_{1}$ and $s_{2}$. We assume that the body, at some instant, occupies the region $B$ with the piecewise smooth surface $\partial B$. The motion of the body is referred to a fixed system of rectangular cartesian axes $O x_{i}$ $(i=1,2,3)$ and to the reference configuration $B$. We denote by $\mathbf{n}$ the outward unit normal of $\partial B$. Letters in boldface stand for tensors of an order $q \geq 1$, and if $\mathbf{v}$ has the order $q$, we write $v_{i j \cdots r}$ ( $q$ subscripts) for the components of $\mathbf{v}$ in the cartesian coordinate frame. We will employ the usual summation and differentiation conventions. Latin subscripts (unless otherwise specified) are understood to range over the integers $(1,2,3)$, summation over repeated subscripts is implied, and subscripts preceded by a comma denote partial differentiation with respect to the corresponding cartesian coordinate. We use a superposed dot to denote the 
partial differentiation with respect to the time $t$. First, we consider the linear theory of elastostatics. We assume that the constituents $s_{1}$ and $s_{2}$ are each elastic solids of Cosserat type. We consider a theory for binary mixtures where the typical particles of $s_{1}$ and $s_{2}$ occupy the same position in the reference configuration. We denote by $\mathbf{u}$ and $\mathbf{w}$ the displacement vector fields associated with the constituents $s_{1}$ and $s_{2}$, respectively. Let $\rho_{\alpha}^{0}$ be the mass density of the constituent $s_{\alpha}$ in the reference configuration. We denote by $\varphi$ and $\psi$ the microrotation vectors associated with the constituents $s_{1}$ and $s_{2}$, respectively. Let $\mathbf{t}$ and $\mathbf{s}$ denote the stress tensors associated with the constituents $s_{1}$ and $s_{2}$, respectively. Further, let $\mathbf{m}$ and $\boldsymbol{\mu}$ denote the partial couplestress tensors associated with $s_{1}$ and $s_{2}$, respectively. The equations of equilibrium can be expressed in the form

$$
\begin{gathered}
t_{j i, j}-p_{i}+\rho_{1}^{0} F_{i}^{(1)}=0, \quad s_{j i, j}+p_{i}+\rho_{2}^{0} F_{i}^{(2)}=0, \\
m_{j i, j}+\frac{1}{2} \varepsilon_{i j k}\left(t_{j k}+s_{j k}\right)-h_{i}+\rho_{1}^{0} G_{i}^{(1)}=0, \\
\mu_{j i, j}+\frac{1}{2} \varepsilon_{i j k}\left(t_{j k}+s_{j k}\right)+h_{i}+\rho_{2}^{0} G_{i}^{(2)}=0,
\end{gathered}
$$

on $B$. Here we have used the following notations: $p_{i}$ is the diffusive force, $h_{i}$ is the diffusive couple, $F_{i}^{(\alpha)}$ is the body force per unit mass acting on the constituent $s_{\alpha}, \varepsilon_{i j k}$ is the alternating symbol, and $G_{i}^{(\alpha)}$ is the body couple per unit mass acting on the constituent $s_{\alpha}$. We introduce the functions $\varepsilon_{i j}$, $e_{i j}, \kappa_{i j}, \gamma_{i j}, d_{i}$, and $\xi_{i}$ by

$$
\begin{gathered}
\varepsilon_{i j}=u_{j, i}+\frac{1}{2} \varepsilon_{i j k}\left(\varphi_{k}+\psi_{k}\right), \quad \kappa_{i j}=\varphi_{j, i}, \\
d_{i}=u_{i}-w_{i}, \\
e_{i j}=w_{j, i}+\frac{1}{2} \varepsilon_{i j k}\left(\varphi_{k}+\psi_{k}\right), \quad \gamma_{i j}=\psi_{j, i}, \\
\xi_{i}=\varphi_{i}-\psi_{i} .
\end{gathered}
$$

The constitutive equations are

$$
\begin{aligned}
& t_{i j}= A_{i j r s} \varepsilon_{r s}+B_{i j r s} \kappa_{r s}+G_{i j r s} e_{r s}+H_{i j r s} \gamma_{r s} \\
&+a_{i j r} d_{r}+g_{i j r} \xi_{r}, \\
& s_{i j}= G_{r s i j} \varepsilon_{r s}+J_{i j r s} \kappa_{r s}+D_{i j r s} e_{r s}+E_{i j r s} \gamma_{r s} \\
&+b_{i j r} d_{r}+h_{i j r} \xi_{r}, \\
& m_{i j}= B_{r s i j} \varepsilon_{r s}+C_{i j r s} \kappa_{r s}+J_{r s i j} e_{r s}+L_{i j r s} \gamma_{r s} \\
&+c_{i j r} d_{r}+\ell_{i j r} \xi_{r}, \\
& \mu_{i j}= H_{i j r s} \varepsilon_{r s}+L_{r s i j} \kappa_{r s}+E_{r s i j} e_{r s}+F_{i j r s} \gamma_{r s} \\
&+f_{i j r} d_{r}+\eta_{i j r} \xi_{r}, \\
& p_{i}=a_{r s i} \varepsilon_{r s}+c_{r s i} \kappa_{r s}+b_{r s i} e_{r s}+f_{r s i} \gamma_{r s}+a_{i j} d_{j}+c_{i j} \xi_{j}, \\
& h_{i}=g_{r s i} \varepsilon_{r s}+e_{r s i} \kappa_{r s}+h_{r s i} e_{r s}+\eta_{r s i} \gamma_{r s}+c_{i j} d_{j}+b_{i j} \xi_{j} .
\end{aligned}
$$

The constitutive coefficients have the following symmetries:

$$
\begin{gathered}
A_{i j r s}=A_{r s i j}, \quad C_{i j r s}=C_{r s i j}, \quad D_{i j r s}=D_{r s i j}, \\
F_{i j r s}=F_{r s i j}, \quad a_{i j}=a_{j i}, \quad b_{i j}=b_{j i} .
\end{gathered}
$$

We assume that (a) $\rho_{1}^{0}$ and $\rho_{2}^{0}$ are strictly positive and continuous on $\bar{B}$; (b) $F_{i}^{(\alpha)}$ and $G_{i}^{(\alpha)},(\alpha=1,2)$, are continuous on $\bar{B}$; (c) the constitutive coefficients are continuously differentiable on $\bar{B}$ and satisfy relations (4).

We say that the array $\omega=\left(u_{i}, w_{i}, \varphi_{i}, \psi_{i}\right)$ is an admissible deformation field on $\bar{B}$ provided $u_{i}, w_{i}, \varphi_{i}, \psi_{i} \in C^{2}(B) \cap C^{1}(\bar{B})$. An admissible stress field on $\bar{B}$ is an ordered array of functions $\left(t_{i j}, s_{i j}, m_{i j}, \mu_{i j}, p_{i}, h_{i}\right)$ with the properties $t_{i j}, s_{i j}, m_{i j}, \mu_{i j}, p_{i}$, $h_{i} \in C^{1}(B) \cap C^{0}(\bar{B})$ and $t_{j i, j}, s_{j i, j}, m_{j i, j}, \mu_{j i, j} \in C^{0}(\bar{B})$. By an admissible state on $\bar{B}$ we mean an ordered array $s=$ $\left(u_{i}, w_{i}, \varphi_{i}, \psi_{i}, \varepsilon_{i j}, e_{i j}, \kappa_{i j}, \gamma_{i j}, d_{i}, \xi_{i}, t_{i j}, s_{i j}, m_{i j}, \mu_{i j}, p_{i}, h_{i}\right)$ with the following properties:

( $\alpha)\left(u_{i}, w_{i}, \varphi_{i}, \psi_{i}\right)$ is an admissible deformation on $\bar{B}$;

(ß) $\varepsilon_{i j}, e_{i j}, \kappa_{i j}, \gamma_{i j}, d_{i}, \xi_{i} \in C^{1}(B) \cap C^{0}(\bar{B})$;

$(\gamma)\left(t_{i j}, s_{i j}, m_{i j}, \mu_{i j}, p_{i}, h_{i}\right)$ is an admissible stress field on $\bar{B}$.

If we define addition and multiplication of an admissible state by a scalar through

$$
\begin{gathered}
s+s^{\prime}=\left(u_{i}+u_{i}^{\prime}, w_{i}+w_{i}^{\prime}, \ldots, h_{i}+h_{i}^{\prime}\right), \\
\lambda s=\left(\lambda u_{i}, \lambda w_{i}, \ldots, \lambda h_{i}\right)
\end{gathered}
$$

then the set of all admissible states is a linear vector space.

We say that $s=\left(u_{i}, w_{i}, \varphi_{i}, \psi_{i}, \varepsilon_{i j}, e_{i j}, \kappa_{i j}, \gamma_{i j}, d_{i}, \xi_{i}, t_{i j}\right.$, $\left.s_{i j}, m_{i j}, \mu_{i j}, p_{i}, h_{i}\right)$ is an elastic state on $B$ corresponding to the body loads $\left(F_{i}^{(1)}, F_{i}^{(2)}, G_{i}^{(1)}, G_{i}^{(2)}\right)$ if $s$ is an admissible state that satisfies (1)-(3) on $B$. Let $S_{r},(r=1,2,3,4)$, be the subsets of $\partial B$ so that $\bar{S}_{1} \cup S_{2}=\bar{S}_{3} \cup S_{4}=\partial B, S_{1} \cap S_{2}=S_{3} \cap S_{4}=\emptyset$. By an external data system on $\bar{B}$ we mean an ordered array $L=\left(F_{i}^{(1)}, F_{i}^{(2)}, G_{i}^{(1)}, G_{i}^{(2)}, \widetilde{u}_{i}, \widetilde{w}_{i}, \widetilde{\varphi}_{i}, \widetilde{\psi}_{i}, \widetilde{t}_{i}, \widetilde{m}_{i}, \widetilde{d}_{i}, \widetilde{\xi}_{i}\right)$ with the following properties: $F_{i}^{(1)}, F_{i}^{(2)}, G_{i}^{(1)}, G_{i}^{(2)} \in C^{0}(\bar{B}) ; \widetilde{u}_{i}, \widetilde{w}_{i} \in$ $C^{0}\left(\bar{S}_{1}\right) ; \tilde{t}_{i}$ are piecewise regular on $S_{2} ; \tilde{t}_{i}$ and $\widetilde{d}_{i}$ are continuous on $S_{2} ; \widetilde{\varphi}_{i}, \widetilde{\psi}_{i} \in C^{0}\left(\bar{S}_{3}\right) ; \widetilde{m}_{i}$ are piecewise regular on $S_{4} ; \widetilde{m}_{i}$ and $\widetilde{d}_{i}$ are continuous on $S_{4}$.

The mixed problem of the equilibrium theory consists in finding an elastic state that corresponds to the body loads $\left(F_{i}^{(1)}, F_{i}^{(2)}, G_{i}^{(1)}, G_{i}^{(2)}\right)$ and satisfies the boundary conditions

$$
\begin{gathered}
u_{i}=\widetilde{u}_{i}, \quad w_{i}=\widetilde{w}_{i} \quad \text { on } \bar{S}_{1}, \\
\left(t_{j i}+s_{j i}\right) n_{j}=\widetilde{t}_{i}, \quad d_{i}=\widetilde{d}_{i} \quad \text { on } S_{2}, \\
\varphi_{i}=\widetilde{\varphi}_{i}, \quad \psi_{i}=\widetilde{\psi}_{i} \quad \text { on } \bar{S}_{3}, \\
\left(m_{j i}+\mu_{j i}\right) n_{j}=\widetilde{m}_{i}, \quad \xi_{i}=\widetilde{\xi}_{i} \quad \text { on } S_{4} .
\end{gathered}
$$

We note that a rigid deformation field is characterized by

$$
u_{i}=w_{i}=A_{i}+\varepsilon_{i j k} B_{j} x_{k}, \quad \varphi_{i}=\psi_{i}=B_{i},
$$

where $A_{i}$ and $B_{i}$ are arbitrary constants. 


\section{Minimum Principle}

In this section we establish a minimum principle of elastostatics which characterize the solution of the mixed problem.

Let $s=\left(u_{i}, w_{i}, \varphi_{i}, \psi_{i}, \varepsilon_{i j}, e_{i j}, \kappa_{i j}, \gamma_{i j}, d_{i}, \xi_{i}, t_{i j}, s_{i j}, m_{i j}, \mu_{i j}\right.$, $p_{i}, h_{i}$ ) be an admissible state on $\bar{B}$. The internal energy density corresponding to $s$ is defined by

$$
\begin{aligned}
W(s)= & \frac{1}{2} A_{i j r s} \varepsilon_{i j} \varepsilon_{r s}+B_{i j r s} \varepsilon_{i j} \kappa_{r s}+\frac{1}{2} C_{i j r s} \kappa_{i j} \kappa_{r s} \\
& +\frac{1}{2} D_{i j r s} e_{i j} e_{r s}+E_{i j r s} e_{i j} \gamma_{r s}+\frac{1}{2} F_{i j r s} \gamma_{i j} \gamma_{r s} \\
& +G_{i j r s} \varepsilon_{i j} e_{r s}+H_{i j r s} \varepsilon_{i j} \gamma_{r s}+J_{i j r s} e_{i j} \kappa_{r s} \\
& +L_{i j r s} \kappa_{i j} \gamma_{r s}+a_{i j r} \varepsilon_{i j} d_{r}+b_{i j r} e_{i j} d_{r}+c_{i j r} \kappa_{i j} d_{r} \\
& +f_{i j r} \gamma_{i j} d_{r}+g_{i j r} \varepsilon_{i j} \xi_{r}+h_{i j r} e_{i j} \xi_{r} \\
& +\ell_{i j r} \kappa_{i j} \xi_{r}+n_{i j r} \gamma_{i j} \xi_{r}+\frac{1}{2} a_{i j} d_{i} d_{j} \\
& +\frac{1}{2} b_{i j} \xi_{i} \xi_{j}+c_{i j} d_{i} \xi_{j} .
\end{aligned}
$$

The strain energy corresponding to $s$ is

$$
U(s)=\int_{B} W(s) d v
$$

By a kinematically admissible state we mean an admissible state that satisfies (2) and the boundary conditions

$$
\begin{gathered}
u_{i}=\tilde{u}_{i}, \quad w_{i}=\widetilde{w}_{i} \quad \text { on } S_{1}, \\
d_{i}=\widetilde{d}_{i} \quad \text { on } S_{2}, \\
\varphi_{i}=\widetilde{\varphi}_{i}, \quad \psi_{i}=\widetilde{\psi}_{i} \quad \text { on } S_{3}, \\
\xi_{i}=\widetilde{\xi}_{i} \quad \text { on } S_{4} .
\end{gathered}
$$

Theorem 1. Assume that the internal energy is a positive definite form. Let $K$ denote the set of all kinematically admissible states, and let $\Lambda(\cdot)$ be the functional on $K$ defined by

$$
\begin{aligned}
\Lambda(s)= & U(s)-\int_{B}\left(\rho_{1}^{0} F_{i}^{(1)} u_{i}+\rho_{2}^{0} F_{i}^{(2)} w_{i}+\rho_{1}^{0} G_{i}^{(1)} \varphi_{i}\right. \\
& \left.+\rho_{2}^{0} G_{i}^{(2)} \psi_{i}\right) d v \\
& -\frac{1}{2} \int_{S_{2}} \widetilde{t}_{i}\left(u_{i}+w_{i}\right) d a-\frac{1}{2} \int_{S_{4}} \widetilde{m}_{i}\left(\varphi_{i}+\psi_{i}\right) d a,
\end{aligned}
$$

for every $s=\left(u_{i}, w_{i}, \varphi_{i}, \psi_{i}, \varepsilon_{i j}, e_{i j}, \kappa_{i j}, \gamma_{i j}, d_{i}, \xi_{i}, t_{i j}, s_{i j}, m_{i j}, \mu_{i j}\right.$, $\left.p_{i}, h_{i}\right) \in K$. Further, let $s$ be a solution of the mixed problem. Then

$$
\Lambda(s) \leq \Lambda\left(s^{*}\right)
$$

for every $s^{*} \in K$, and equality holds only if $s^{*}=s$ modulo a rigid displacement.
Proof. Let $s, s^{*} \in K$ and define

$$
s^{\prime}=s^{*}-s .
$$

Then $s^{\prime}=\left(u_{i}^{\prime}, w_{i}^{\prime}, \varphi_{i}^{\prime}, \psi_{i}^{\prime}, \varepsilon_{i j}^{\prime}, e_{i j}^{\prime}, \kappa_{i j}^{\prime}, \gamma_{i j}^{\prime}, d_{i}^{\prime}, \xi_{i}^{\prime}, t_{i j}^{\prime}, s_{i j}^{\prime}, m_{i j}^{\prime}, \mu_{i j}^{\prime}\right.$, $\left.p_{i}^{\prime}, h_{i}^{\prime}\right)$ is an admissible state with the properties

$$
\begin{aligned}
\varepsilon_{i j}^{\prime} & =u_{j, i}^{\prime}+\frac{1}{2} \varepsilon_{i j k}\left(\varphi_{k}^{\prime}+\psi_{k}^{\prime}\right), \\
\kappa_{i j}^{\prime} & =\varphi_{j, i}^{\prime}, \quad d_{i}^{\prime}=u_{i}^{\prime}-w_{i}^{\prime}, \\
e_{i j}^{\prime} & =w_{j, i}^{\prime}+\frac{1}{2} \varepsilon_{i j k}\left(\varphi_{k}^{\prime}+\psi_{k}^{\prime}\right), \\
\gamma_{i j}^{\prime} & =\psi_{j, i}^{\prime}, \quad \xi_{i}^{\prime}=\varphi_{i}^{\prime}-\psi_{i}^{\prime}, \\
w_{i}^{\prime} & =0 \quad \text { on } S_{1}, \quad d_{i}^{\prime}=0 \quad \text { on } S_{2}, \\
u_{i}^{\prime}=0, \quad \psi_{i}^{\prime} & =0 \quad \text { on } S_{3}, \quad \xi_{i}^{\prime}=0 \quad \text { on } S_{4} .
\end{aligned}
$$

It follows from (4), (8), (9), (11), and (13) that

$$
\begin{aligned}
\Lambda\left(s^{*}\right)= & \Lambda(s)+E\left(s^{\prime}\right) \\
& +\int_{B}\left(t_{i j} \varepsilon_{i j}^{\prime}+s_{i j} e_{i j}^{\prime}+m_{i j} \kappa_{i j}^{\prime}+\mu_{i j} \gamma_{i j}^{\prime}+p_{i} d_{i}^{\prime}+h_{i} \xi_{i}^{\prime}\right) d v \\
& -\int_{B}\left(\rho_{1}^{0} F_{i}^{(1)} u_{i}^{\prime}+\rho_{2}^{0} F_{i}^{(2)} w_{i}^{\prime}+\rho_{1}^{0} G_{i}^{(1)} \varphi_{i}^{\prime}\right. \\
& \left.+\rho_{2}^{0} G_{i}^{(2)} \psi_{i}^{\prime}\right) d v \\
& -\frac{1}{2} \int_{S_{i}} \widetilde{t}_{i}\left(u_{i}^{\prime}+w_{i}^{\prime}\right) d a-\frac{1}{2} \int_{S_{4}} \widetilde{m}_{i}\left(\varphi_{i}^{\prime}+\psi_{i}^{\prime}\right) d a .
\end{aligned}
$$

If we take into account (14) and use (1), then we obtain

$$
\begin{aligned}
t_{i j} \varepsilon_{i j}^{\prime}+ & s_{i j} e_{i j}^{\prime}+m_{i j} \kappa_{i j}^{\prime}+\mu_{i j} \gamma_{i j}^{\prime}+p_{i} d_{i}^{\prime}+h_{i} \xi_{i}^{\prime} \\
= & \left(t_{j i} u_{i}^{\prime}+s_{j i} w_{i}^{\prime}+m_{j i} \varphi_{i}^{\prime}+\mu_{j i} \psi_{i}^{\prime}\right)_{, j} \\
& -\left(t_{j i, j}-p_{i}\right) u_{i}^{\prime}-\left(s_{j i, j}+p_{i}\right) w_{i}^{\prime} \\
& -\left[m_{j i, j}+\frac{1}{2} \varepsilon_{i r k}\left(t_{r k}+s_{r k}\right)-h_{i}\right] \varphi_{i}^{\prime} \\
& -\left[\mu_{j i, j}+\frac{1}{2} \varepsilon_{i r j}\left(t_{r j}+s_{r j}\right)+h_{i}\right] \psi_{i}^{\prime} \\
= & \left(t_{j i} u_{i}^{\prime}+s_{j i} w_{i}^{\prime}+m_{j i} \varphi_{i}^{\prime}+\mu_{j i} \psi_{i}^{\prime}\right)_{, j} \\
& +\rho_{1}^{0} F_{i}^{(1)} u_{i}^{\prime}+\rho_{2}^{0} F_{i}^{(2)} w_{i}^{\prime}+\rho_{1}^{0} G_{i}^{(1)} \varphi_{i}^{\prime}+\rho_{2}^{0} G_{i}^{(2)} \psi_{i}^{\prime} .
\end{aligned}
$$


By using the divergence theorem, (15), and (17) we get

$$
\begin{aligned}
& \int_{B}\left(t_{j i} \varepsilon_{i j}^{\prime}+s_{i j} e_{i j}^{\prime}+m_{i j} \kappa_{i j}^{\prime}+\mu_{i j} \gamma_{i j}^{\prime}+p_{i} d_{i}^{\prime}+h_{i} \xi_{i}^{\prime}\right) d v \\
& =\frac{1}{2} \int_{\partial B}\left[\left(t_{j i}+s_{j i}\right) n_{j}\left(u_{i}^{\prime}+w_{i}^{\prime}\right)+\left(t_{j i}-s_{j i}\right) n_{j} d_{i}^{\prime}\right. \\
& \left.\quad+\left(m_{j i}+\mu_{j i}\right) n_{j}\left(\varphi_{i}^{\prime}+\psi_{i}^{\prime}\right)+\left(m_{j i}-\mu_{j i}\right) \xi_{i}^{\prime}\right] d a \\
& +\int_{B}\left(\rho_{1}^{0} F_{i}^{(1)} u_{i}^{\prime}+\rho_{2}^{0} F_{i}^{(2)} w_{i}^{\prime}+\rho_{1}^{0} G_{i}^{(1)} \varphi_{i}^{\prime}+\rho_{2}^{0} G_{i}^{(2)} \psi_{i}^{\prime}\right) d v \\
& =\frac{1}{2} \int_{S_{2}}\left(t_{j i}+s_{j i}\right) n_{j}\left(u_{i}^{\prime}+w_{i}^{\prime}\right) d a \\
& \quad+\frac{1}{2} \int_{S_{4}}\left(m_{j i}+\mu_{j i}\right) n_{j}\left(\varphi_{i}^{\prime}+\psi_{i}^{\prime}\right) d a \\
& \quad+\int_{B}\left(\rho_{1}^{0} F_{i}^{(1)} u_{i}^{\prime}+\rho_{2}^{0} F_{i}^{(2)} w_{i}^{\prime}+\rho_{1}^{0} G_{i}^{(1)} \varphi_{i}^{\prime}+\rho_{2}^{0} G_{i}^{(2)} \psi_{i}^{\prime}\right) d v .
\end{aligned}
$$

In view of (18), relation (16) can be written as

$$
\begin{aligned}
\Lambda\left(s^{*}\right)= & \Lambda(s)+E\left(s^{\prime}\right) \\
& +\frac{1}{2} \int_{S_{2}}\left[\left(t_{j i}+s_{j i}\right) n_{j}-\widetilde{t}_{i}\right]\left(u_{i}^{\prime}+w_{i}^{\prime}\right) d a \\
& +\frac{1}{2} \int_{S_{4}}\left[\left(m_{j i}+\mu_{j i}\right) n_{j}-\widetilde{m}_{i}\right]\left(\varphi_{i}^{\prime}+\psi_{i}^{\prime}\right) d a .
\end{aligned}
$$

Since $s$ is a solution of the mixed problem, (19) implies

$$
\Lambda\left(s^{*}\right)-\Lambda(s)=E\left(s^{\prime}\right) .
$$

Since the internal energy is positive definite, we obtain

$$
\Lambda(s) \leq \Lambda\left(s^{*}\right) .
$$

Moreover, $\Lambda(s)=\Lambda\left(s^{*}\right)$ only if $\varepsilon_{i j}^{\prime}=0, e_{i j}^{\prime}=0, \kappa_{i j}^{\prime}=0$, $\gamma_{i j}^{\prime}=0, d_{i}^{\prime}=0$, and $\xi_{i}^{\prime}=0$. Thus, $\Lambda(s)=\Lambda\left(s^{*}\right)$ only if $s^{*}=s$ modulo a rigid displacement.

Theorem 1 extends the principle of minimum potential energy from the classical elasticity (see, e.g., [16]).

\section{Dynamic Theory}

In this section we establish a minimum principle of Reiss type (see $[17,18])$ in the dynamic theory. The equations of motions are

$$
\begin{gathered}
t_{j i, j}-p_{i}+\rho_{1}^{0} F_{i}^{(1)}=\rho_{1} \ddot{u}_{i}, \\
s_{j i, j}+p_{i}+\rho_{2}^{0} F_{i}^{(2)}=\rho_{2}^{0} \ddot{w}_{i}, \\
m_{j i, j}+\frac{1}{2} \varepsilon_{i j k}\left(t_{j k}+s_{j k}\right)-h_{i}+\rho_{1}^{0} G_{i}^{(1)}=J_{i j}^{(1)} \ddot{\varphi}_{j}, \\
\mu_{j i, j}+\frac{1}{2} \varepsilon_{i j k}\left(t_{j k}+s_{j k}\right)+h_{i}+\rho_{2}^{0} G_{i}^{(2)}=J_{i j}^{(2)} \ddot{\varphi}_{j},
\end{gathered}
$$

on $B \times I$, where $J_{i j}^{(1)}$ and $J_{i j}^{(2)}$ are coefficients of inertia and $I=(0, \infty)$. To the field equations (22), (2), and (3) we must add boundary conditions and initial conditions. The initial conditions are

$$
\begin{array}{ll}
u_{i}(\mathbf{x}, 0)=u_{i}^{0}(\mathbf{x}), & \dot{u}_{i}(\mathbf{x}, 0)=v_{i}^{0}(\mathbf{x}), \\
w_{i}(\mathbf{x}, 0)=w_{i}^{0}(\mathbf{x}), & \dot{w}_{i}(\mathbf{x}, 0)=\omega_{i}^{0}(\mathbf{x}), \\
\varphi_{i}(\mathbf{x}, 0)=\varphi_{i}^{0}(\mathbf{x}), & \dot{\varphi}_{i}(\mathbf{x}, 0)=\zeta_{i}^{0}(\mathbf{x}), \\
\psi_{i}(\mathbf{x}, 0)=\psi_{i}^{0}(\mathbf{x}), & \dot{\psi}_{i}(\mathbf{x}, 0)=\chi_{i}^{0}(\mathbf{x}),
\end{array}
$$

where $u_{i}^{0}, v_{i}^{0}, w_{i}^{0}, \omega_{i}^{0}, \varphi_{i}^{0}, \zeta_{i}^{0}, \psi_{i}^{0}$, and $\chi_{i}^{0}$ are prescribed functions. We consider the boundary conditions

$$
\begin{gathered}
u_{i}=\widetilde{u}_{i}, \quad w_{i}=\widetilde{w}_{i} \quad \text { on } \bar{S}_{1} \times I, \\
\left(t_{j i}+s_{j i}\right) n_{j}=\widetilde{t}_{i}, \quad d_{i}=\widetilde{d}_{i} \quad \text { on } S_{2} \times I, \\
\varphi_{i}=\widetilde{\varphi}_{i}, \quad \psi_{i}=\widetilde{\psi}_{i} \quad \text { on } \bar{S}_{3} \times I, \\
\left(m_{j i}+\mu_{j i}\right) n_{j}=\widetilde{m}_{i}, \quad \xi_{i}=\widetilde{\xi}_{i} \quad \text { on } S_{4} \times I,
\end{gathered}
$$

where $\widetilde{u}_{i}, \widetilde{w}_{i}, \widetilde{t}_{i}, \widetilde{d}_{i}, \widetilde{\varphi}_{i}, \widetilde{\psi}_{i}, \widetilde{m}_{i}$, and $\widetilde{\xi}_{i}$ are given.

We assume that

(i) $F_{i}^{(1)}, F_{i}^{(2)}, G_{i}^{(1)}$, and $G_{i}^{(2)}$ are continuous on $B \times[0, \infty)$;

(ii) $\rho_{1}^{0}, \rho_{2}^{0}, J_{i j}^{(1)}, J_{i j}^{(2)}, u_{i}^{0}, v_{i}^{0}, w_{i}^{0}, \omega_{i}^{0}, \varphi_{i}^{0}, \zeta_{i}^{0}, \psi_{i}^{0}$, and $\chi_{i}^{0}$ are continuous on $\bar{B}$;

(iii) the constitutive coefficients are continuously differentiable on $\bar{B}$;

(iv) the constitutive coefficients satisfy relations (4), and the coefficients of inertia are symmetric;

(v) $\rho_{1}^{0}$ and $\rho_{2}^{0}$ are strictly positive on $\bar{B}$, and $J_{i j}^{(1)}$ and $J_{i j}^{(2)}$ are positive definite tensors on $\bar{B}$;

(vi) $\widetilde{u}_{i}, \widetilde{w}_{i}$ are continuous on $\bar{S}_{1} \times I$, and $\widetilde{\varphi}_{i}, \widetilde{\psi}_{i}$ are continuous on $\bar{S}_{3} \times I$;

(vii) $\tilde{d}_{i}$ and $\tilde{\xi}_{i}$ are continuous on $S_{2} \times I$ and $S_{4} \times I$, respectively;

(viii) $\widetilde{t}_{i}$ and $\widetilde{m}_{i}$ are continuous in time and piecewise regular on $S_{2} \times I$ and $S_{4} \times I$, respectively.

Let $M$ and $N$ bo nonnegative integers. We say that $F$ is of class $C^{M, N}$ on $B \times I$ if $F$ is continuous on $B \times I$ and the functions

$$
\begin{gathered}
\frac{\partial^{m}}{\partial x_{i} \partial x_{j} \cdots \partial x_{r}}\left(\frac{\partial^{n} F}{\partial t^{n}}\right), \\
m \in\{0,1,2, \ldots, M\}, \quad n \in\{0,1,2, \ldots, N\}, \\
m+n \leq \max \{M, N\}
\end{gathered}
$$

exist and are continuous on $B \times I$. We denote $C^{M, N}$ by $C^{M}$.

We say that $\omega=\left(u_{i}, w_{i}, \varphi_{i}, \psi_{i}\right)$ is a dynamically admissible deformation field on $\bar{B} \times I$ provided that $(\alpha) u_{i}, w_{i}, \varphi_{i}, \psi_{i} \in$ 
$C^{2}(B) \cap C^{1}(\bar{B}) ;(\beta) u_{i}, \dot{u}_{i}, u_{i, j}, w_{i}, \dot{w}_{i}, w_{i, j}, \varphi_{i}, \dot{\varphi}_{i}, \varphi_{i, j}, \psi_{i}, \dot{\psi}_{i}, \psi_{i, j}$ are continuous on $\bar{B} \times[0, \infty)$. By a dynamically admissible stress field on $\bar{B} \times I$ we mean an ordered array of functions $\left(t_{i j}, s_{i j}, m_{i j}, \mu_{i j}, p_{i}, h_{i}\right)$ with the following properties: (a) $t_{i j}, s_{i j}, m_{i j}$, and $\mu_{i j}$ are of class $C^{1,0}$ on $B \times I$; (b) $p_{i}, h_{i} \in C^{0}$ on $B \times I$; (c) $t_{i j}, t_{j i, j}, s_{i j}, s_{j i, j}, m_{i j}, m_{j i, j}, \mu_{i j}, \mu_{j i, j}, p_{i}$, and $h_{i}$ are continuous on $\bar{B} \times[0, \infty)$.

An admissible process on $\bar{B} \times I$ is an ordered array $P=$ $\left(u_{i}, w_{i}, \varphi_{i}, \psi_{i}, \varepsilon_{i j}, e_{i j}, \kappa_{i j}, \gamma_{i j}, d_{i}, \xi_{i}, t_{i j}, s_{i j}, m_{i j}, \mu_{i j}, p_{i}, h_{i}\right)$ with the following properties:

(1) $\left(u_{i}, w_{i}, \varphi_{i}, \psi_{i}\right)$ is a dynamically admissible deformation field;

(2) $\varepsilon_{i j}, e_{i j}, \kappa_{i j}, \gamma_{i j}, d_{i}, \xi_{i} \in C^{1,0}$ on $B \times I$;

(3) $\varepsilon_{i j}, e_{i j}, \kappa_{i j}, \gamma_{i j}, d_{i}$, and $\xi_{i}$ are continuous on $\bar{B} \times[0, \infty)$;

(4) $\left(t_{i j}, s_{i j}, m_{i j}, \mu_{i j}, p_{i}, h_{i}\right)$ is a dynamically admissible stress field on $\bar{B} \times I$.

We say that $P=\left(u_{i}, w_{i}, \varphi_{i}, \psi_{i}, \varepsilon_{i j}, e_{i j}, \kappa_{i j}, \gamma_{i j}, d_{i}, \xi_{i}, t_{i j}\right.$, $\left.s_{i j}, m_{i j}, \mu_{i j}, p_{i}, h_{i}\right)$ is an elastic process corresponding to the body loads $\left(F_{i}^{(1)}, F_{i}^{(2)}, G_{i}^{(1)}, G_{i}^{(2)}\right)$ if $P$ is an admissible process that satisfies (22), (2), and (3) on $B \times I$. Given an external data system $\left(F_{i}^{(1)}, F_{i}^{(2)}, G_{i}^{(1)}, G_{i}^{(2)}, \widetilde{u}_{i}, \widetilde{w}_{i}, \widetilde{\varphi}_{i}, \widetilde{\psi}_{i}, \widetilde{t}_{i}, \widetilde{m}_{i}\right.$, $\left.\widetilde{d}_{i}, \widetilde{\xi}_{i}, u_{i}^{0}, v_{i}^{0}, w_{i}^{0}, \omega_{i}^{0}, \varphi_{i}^{0}, \zeta_{i}^{0}, \psi_{i}^{0}, \chi_{i}^{0}\right)$ on $\bar{B} \times I$, the dynamic problem consists in finding an elastic process corresponding to the body loads $\left(F_{i}^{(1)}, F_{i}^{(2)}, G_{i}^{(1)}, G_{i}^{(2)}\right)$ which satisfies the initial conditions (23) and the boundary conditions (24). We call such an elastic process a solution of the mixed problem.

Let $k$ be the function defined by

$$
k(t)=t, \quad t \in[0, \infty) .
$$

Let $F$ and $G$ be functions on $B \times I$ that are continuous in time. We denote by $F * G$ the convolution of $F$ and $G$ :

$$
[F * G](\mathbf{x}, t)=\int_{0}^{t} F(\mathbf{x}, t-\tau) G(\mathbf{x}, \tau) d \tau, \quad \mathbf{x} \in B, t \in I .
$$

We define the functions $f_{i}^{(1)}, f_{i}^{(2)}, g_{i}^{(1)}$, and $g_{i}^{(2)}$ on $\bar{B} \times$ $[0, \infty)$ by

$$
\begin{aligned}
& f_{i}^{(1)}=\rho_{1}^{0} k * F_{i}^{(1)}+\rho_{1}^{0}\left(t v_{i}^{0}+u_{i}^{0}\right), \\
& f_{i}^{(2)}=\rho_{2}^{0} k * F_{i}^{(2)}+\rho_{2}^{0}\left(t \omega_{i}^{0}+w_{i}^{0}\right), \\
& g_{i}^{(1)}=\rho_{1}^{0} k * G_{i}^{(1)}+\rho_{1}^{0}\left(t \zeta_{i}^{0}+\varphi_{i}^{0}\right), \\
& g_{i}^{(2)}=\rho_{2}^{0} k * G_{i}^{(2)}+\rho_{2}^{0}\left(t \chi_{i}^{0}+\psi_{i}^{0}\right) .
\end{aligned}
$$

Following [16] we can prove the following.
Theorem 2. Let $u_{i}, w_{i}, \varphi_{i}, \psi_{i} \in C^{0,2}, t_{i j}, s_{i j}, m_{i j}, \mu_{i j} \in C^{1,0}$, $p_{i}, h_{i} \in C^{0}$. Then $u_{i}, w_{i}, \varphi_{i}, \psi_{i}, t_{i j}, s_{i j}, m_{i j}, \mu_{i j}, p_{i}$, and $h_{i}$ satisfy (22) and the initial conditions (23) if and only if

$$
\begin{gathered}
k *\left(t_{j i, j}-p_{i}\right)+f_{i}^{(1)}=\rho_{1}^{0} u_{i}, \\
k *\left(s_{j i, j}+p_{i}\right)+f_{i}^{(2)}=\rho_{2}^{0} w_{i}, \\
k *\left[m_{j i, j}+\frac{1}{2} \varepsilon_{i j k}\left(t_{j k}+s_{j k}\right)-h_{i}\right]+g_{i}^{(1)}=J_{i j}^{(1)} \varphi_{j}, \\
k *\left[\mu_{j i, j}+\frac{1}{2} \varepsilon_{i j k}\left(t_{j k}+s_{j k}\right)+h_{i}\right]+g_{i}^{(2)} \\
=J_{i j}^{(2)} \psi_{j} \quad \text { on } B \times[0, \infty) .
\end{gathered}
$$

The next theorem gives an alternative characterization of the mixed problem in which the initial conditions are incorporated into the field equations. This result is useful in the derivation of minimum principle.

Theorem 3. Let $P$ be an admissible process. Then $P$ is a solution of the mixed problem if and only if $P$ satisfies (2), (3), and (29) on $B \times[0, \infty)$ and the boundary conditions (24).

In what follows we denote by $\widehat{H}$ or $\mathscr{L} H$ the Laplace transform with respect to the time of the function $H$ :

$$
\widehat{H}(p)=(\mathscr{L} H)(p)=\int_{0}^{\infty} e^{-p t} H(t) d t, \quad\left(p_{0} \leq p<\infty\right),
$$

for some $p_{0} \geq 0$.

In addition to the assumptions made previously, we assume that

$$
\begin{aligned}
& \left(A_{1}\right) F_{i}^{(1)}, F_{i}^{(2)}, G_{i}^{(1)}, G_{i}^{(2)}, \widetilde{u}_{i}, \widetilde{w}_{i}, \widetilde{t}_{i}, \widetilde{d}_{i}, \widetilde{\varphi}_{i}, \widetilde{\psi}_{i}, \widetilde{m}_{i} \text {, and } \widetilde{\xi}_{i} \text { pos- } \\
& \text { sess Laplace transforms; } \\
& \left(A_{2}\right) \text { the internal energy } W \text { is positive for any } \\
& \varepsilon_{i j}, e_{i j}, \kappa_{i j}, \gamma_{i j}, d_{i} \text {, and } \xi_{i} .
\end{aligned}
$$

We say that $f$ is bounded at infinity if $\lim _{t \rightarrow \infty} f(\mathbf{x}, t)$ exists for each $\mathbf{x}$ in the domain of definition of $f$. We will assume that the functions used to describe the mixed problem are bounded at infinity.

We write $f^{[n]}$ for the $n$th derivative of $f$ with respect to $t$ holding $\mathbf{x}$ fixed. Following [17] we introduce the set $\Gamma$ of admissible weight functions. We say that $g \in \Gamma$ if $g$ is a function on $[0, \infty)$ with the following properties:

$(\alpha) \int_{0}^{\infty} \int_{0}^{\infty} g^{[k]}(t+s) d t d s$ exists for $k \geq 0$;

(ß) $g(t)=\int_{0}^{\infty} G(p) e^{-p t} d p, t \in[0, \infty)$,

where $G$ is a continuous and positive function on $[0, \infty)$ and has a finite limit at infinity. An example of a weight function is $g(t)=(t+2)^{-3}$ with $2 G(t)=t^{2} \exp (-2 t)$ (cf. [17]). 
By a kinematically admissible process we mean an admissible process that satisfies (2) and the boundary conditions

$$
\begin{gathered}
u_{i}=\tilde{u}_{i}, \quad w_{i}=\widetilde{w}_{i} \quad \text { on } \bar{S}_{1} \times I, \\
d_{i}=\widetilde{d}_{i} \quad \text { on } S_{2} \times I, \\
\varphi_{i}=\widetilde{\varphi}_{i}, \quad \psi_{i}=\widetilde{\psi}_{i} \quad \text { on } \bar{S}_{3} \times I, \\
\xi_{i}=\tilde{\xi}_{i} \quad \text { on } S_{4} \times I .
\end{gathered}
$$

Let $\mathscr{K}$ denote the set of all kinematically admissible processes $P$ such that the functions of $P$ and their first derivatives with respect to cartesian coordinates possess Laplace transforms.

Theorem 4. Assume that hypotheses $\left(A_{1}\right)$ and $\left(A_{2}\right)$ hold. Let $\Phi_{g}(\cdot)$ be the functional on $\mathscr{K}$ defined by

$$
\begin{aligned}
& \Phi_{g}(P)=\int_{B} \int_{0}^{\infty} \int_{0}^{\infty} g(t+s) \\
& \cdot\left\{\left(A_{i j m n} k * \varepsilon_{m n}\right)(\mathbf{x}, t) \varepsilon_{i j}(\mathbf{x}, s)\right. \\
& +2\left(B_{i j m n} k * \kappa_{m n}\right)(\mathbf{x}, t) \varepsilon_{i j}(\mathbf{x}, s) \\
& +\left(C_{i j m n} k * \kappa_{m n}\right)(\mathbf{x}, t) \kappa_{i j}(\mathbf{x}, s) \\
& +\left(D_{i j m n} k * e_{m n}\right)(\mathbf{x}, t) e_{i j}(\mathbf{x}, s) \\
& +2\left(E_{i j m n} k * \gamma_{m n}\right)(\mathbf{x}, t) e_{i j}(\mathbf{x}, s) \\
& +\left(F_{i j m n} k * \gamma_{m n}\right)(\mathbf{x}, t) \gamma_{i j}(\mathbf{x}, s) \\
& +2\left(G_{i j m n} k * e_{m n}\right)(\mathbf{x}, t) \varepsilon_{i j}(\mathbf{x}, s) \\
& +2\left(H_{i j m n} k * \gamma_{m n}\right)(\mathbf{x}, t) \varepsilon_{i j}(\mathbf{x}, s) \\
& +2\left(J_{i j m n} k * \kappa_{m n}\right)(\mathbf{x}, t) e_{i j}(\mathbf{x}, s) \\
& +2\left(L_{i j m n} k * \gamma_{m n}\right)(\mathbf{x}, t) \kappa_{i j}(\mathbf{x}, s) \\
& +2\left(a_{i j r} k * d_{r}\right)(\mathbf{x}, t) \varepsilon_{i j}(\mathbf{x}, s) \\
& +2\left(b_{i j m} k * d_{m}\right)(\mathbf{x}, t) e_{i j}(\mathbf{x}, s) \\
& +2\left(c_{i j m} k * d_{m}\right)(\mathbf{x}, t) \kappa_{i j}(\mathbf{x}, s) \\
& +2\left(f_{i j m} k * d_{m}\right)(\mathbf{x}, t) \gamma_{i j}(\mathbf{x}, s) \\
& +2\left(g_{i j m} k * \xi_{m}\right)(\mathbf{x}, t) \varepsilon_{i j}(\mathbf{x}, s) \\
& +2\left(h_{i j m} k * \xi_{m}\right)(\mathbf{x}, t) e_{i j}(\mathbf{x}, s) \\
& +2\left(\ell_{i j m} k * \xi_{m}\right)(\mathbf{x}, t) \kappa_{i j}(\mathbf{x}, s) \\
& +2\left(n_{i j m} k * \xi_{m}\right)(\mathbf{x}, t) \gamma_{i j}(\mathbf{x}, s)
\end{aligned}
$$

$$
\begin{aligned}
& +\left(a_{i j} k * d_{j}\right)(\mathbf{x}, t) d_{i}(\mathbf{x}, s) \\
& +\left(b_{i j} k * \xi_{j}\right)(\mathbf{x}, t) \xi_{i}(\mathbf{x}, s) \\
& +2\left(c_{i j} k * \xi_{j}\right)(\mathbf{x}, t) d_{i}(\mathbf{x}, s) \\
& +\left(\rho_{1}^{0} u_{i}\right)(\mathbf{x}, t) u_{i}(\mathbf{x}, s) \\
& +\left(\rho_{2}^{0} w_{i}\right)(\mathbf{x}, t) w_{i}(\mathbf{x}, s) \\
& +\left(J_{i j}^{(1)} \varphi_{j}\right)(\mathbf{x}, t) \varphi_{i}(\mathbf{x}, s) \\
& \left.+\left(J_{i j}^{(2)} \psi_{j}\right)(\mathbf{x}, t) \psi_{i}(\mathbf{x}, s)\right\} d t d s d v_{x} \\
& -2 \int_{B} \int_{0}^{\infty} \int_{0}^{\infty} g(t+s) \\
& \cdot\left[f_{i}^{(1)}(\mathbf{x}, t) u_{i}(\mathbf{x}, s)+f_{i}^{(2)}(\mathbf{x}, t) w_{i}(\mathbf{x}, s)\right. \\
& +g_{i}^{(1)}(\mathbf{x}, t) \varphi_{i}(\mathbf{x}, s) \\
& \left.+g_{i}^{(2)}(\mathbf{x}, t) \psi_{i}(\mathbf{x}, s)\right] d t d s d v_{x} \\
& -\int_{S_{2}} \int_{0}^{\infty} \int_{0}^{\infty} g(t+s)\left(k * \tilde{t}_{i}\right)(\mathbf{x}, t)\left(u_{i}+w_{i}\right) \\
& \cdot(\mathbf{x}, s) d t d s d a_{x} \\
& -\int_{S_{4}} \int_{0}^{\infty} \int_{0}^{\infty} g(t+s)\left(k * \widetilde{m}_{i}\right)(\mathbf{x}, t)\left(\varphi_{i}+\psi_{i}\right) \\
& \cdot(\mathbf{x}, s) d t d s d a_{x},
\end{aligned}
$$

for every $P \in \mathscr{K}$. Further, let $P$ be a solution of the mixed problem. Then

$$
\Phi_{g}(P) \leq \Phi_{g}\left(P^{*}\right)
$$

for every $P^{*} \in \mathscr{K}$.

Proof. We consider $P, P^{*} \in \mathscr{K}$ and introduce $P^{\prime}$ by $P^{\prime}=$ $P^{*}-P$. If we denote $P^{\prime}=\left(u_{i}^{\prime}, w_{i}^{\prime}, \varphi_{i}^{\prime}, \psi_{i}^{\prime}, \varepsilon_{i j}^{\prime}, e_{i j}^{\prime}, \kappa_{i j}^{\prime}, \gamma_{i j}^{\prime}, d_{i}^{\prime}, \xi_{i}^{\prime}\right.$, $\left.t_{i j}^{\prime}, s_{i j}^{\prime}, \mu_{i j}^{\prime}, p_{i}^{\prime}, h_{i}^{\prime}\right)$ then we have

$$
\begin{gathered}
2 \varepsilon_{i j}^{\prime}=u_{j, i}^{\prime}+\varepsilon_{i j k}\left(\varphi_{k}^{\prime}+\psi_{k}^{\prime}\right), \\
\kappa_{i j}^{\prime}=\varphi_{j, i}^{\prime}, \quad d_{i}^{\prime}=u_{i}^{\prime}-w_{i}^{\prime}, \\
2 e_{i j}^{\prime}=w_{j, i}^{\prime}+\varepsilon_{i j k}\left(\varphi_{k}^{\prime}+\psi_{k}^{\prime}\right), \\
\gamma_{i j}^{\prime}=\psi_{j, i}^{\prime} \quad \xi_{i}^{\prime}=\varphi_{i}^{\prime}-\psi_{i}^{\prime}, \\
u_{i}^{\prime}=0, \quad w_{i}^{\prime}=0 \quad \text { on } S_{2}, \\
\varphi_{i}^{\prime}=0, \quad \psi_{i}^{\prime}=0 \quad \text { on } S_{3}, \quad \xi_{i}^{\prime}=0 \quad \text { on } S_{4} .
\end{gathered}
$$

We note that

$$
\mathscr{L}(M * N)=\widehat{M} \widehat{N}, \quad(\mathscr{L} k)(p)=p^{-2}
$$


If we use the definition of the weight functions we get

$$
\begin{gathered}
\int_{B} \int_{0}^{\infty} \int_{0}^{\infty} g(t+s) H(\mathbf{x}, t) Q(\mathbf{x}, s) d t d s d v_{x} \\
=\int_{B} \int_{0}^{\infty} G(P) \widehat{H}(\mathbf{x}, p) \widehat{Q}(\mathbf{x}, p) d p d v_{x} .
\end{gathered}
$$

By using (35) and (36) the functional $\Phi_{g}(\cdot)$ can be expressed in the form

$$
\begin{aligned}
& \Phi_{g}(P)=\int_{B} \int_{0}^{\infty} p^{-2} G(p) \\
& \cdot\left[A_{i j m n} \widehat{\varepsilon}_{m n} \widehat{\varepsilon}_{i j}+2 B_{i j m n} \widehat{\kappa}_{m n} \widehat{\varepsilon}_{i j}\right. \\
& +C_{i j m n} \widehat{\kappa}_{m n} \widehat{\kappa}_{i j}+D_{i j m n} \widehat{e}_{m n} \widehat{e}_{i j} \\
& +2 E_{i j m n} \widehat{\gamma}_{m n} \widehat{e}_{i j}+F_{i j m n} \widehat{\gamma}_{m n} \widehat{\gamma}_{i j} \\
& +2 G_{i j m n} \widehat{e}_{m n} \widehat{\varepsilon}_{i j}+2 H_{i j m n} \widehat{\gamma}_{m n} \widehat{\varepsilon}_{i j} \\
& +2 J_{i j m n} \widehat{\kappa}_{m n} \widehat{\varepsilon}_{i j}+2 L_{i j m n} \widehat{\gamma}_{m n} \widehat{\varepsilon}_{i j} \\
& +2 a_{i j m} \widehat{d}_{m} \widehat{\varepsilon}_{i j}+2 b_{i j m} \widehat{d}_{m} \widehat{e}_{i j} \\
& +2 c_{i j m} \widehat{d}_{m} \widehat{\kappa}_{i j}+2 f_{i j m} \widehat{d}_{m} \widehat{\gamma}_{i j} \\
& +2 g_{i j m} \widehat{\xi}_{m} \widehat{\varepsilon}_{i j}+2 h_{i j m} \widehat{\xi}_{m} \widehat{e}_{i j} \\
& +2 \ell_{i j m} \widehat{\xi}_{m} \widehat{\kappa}_{i j}+2 n_{i j m} \widehat{\xi}_{m} \widehat{\gamma}_{i j} \\
& +a_{i j} \widehat{d}_{j} \widehat{d}_{i}+b_{i j} \widehat{\xi}_{j} \widehat{\xi}_{i}+2 c_{i j} \widehat{\xi}_{j} \widehat{d}_{i} \\
& +p^{2}\left(\rho_{1}^{0} \widehat{u}_{i} \widehat{u}_{i}+\rho_{2}^{0} \widehat{w}_{i} \widehat{w}_{i}+J_{i j}^{(1)} \widehat{\varphi}_{j} \widehat{\varphi}_{i}\right. \\
& \left.\left.+J_{i j}^{(2)} \widehat{\psi}_{j} \widehat{\psi}_{i}\right)\right] d p d v_{x} \\
& -2 \int_{B} \int_{0}^{\infty} G(p)\left[\widehat{f}_{i}^{(1)} \widehat{u}_{i}+\widehat{f}_{i}^{(2)} \widehat{w}_{i}+\widehat{g}_{i}^{(1)} \widehat{\varphi}_{i}+\widehat{g}_{i}^{(2)} \widehat{\psi}_{i}\right] d p d v_{x} \\
& -\int_{S_{2}} \int_{0}^{\infty} p^{-2} G(p)\left(\widehat{u}_{i}+\widehat{w}_{i}\right)\left(\mathscr{L} \widetilde{t}_{i}\right) d p d a_{x} \\
& -\int_{S_{4}} \int_{0}^{\infty} G(p)\left(\widehat{\varphi}_{i}+\widehat{\psi}_{i}\right)\left(\mathscr{L} \widetilde{m}_{i}\right) d p d a_{x},
\end{aligned}
$$

where, for convenience, we have suppressed the argument $\mathbf{x}$.

If we take the Laplace transform of relations (29) and use (35) we get

$$
\begin{gathered}
p^{-2}\left(\widehat{t}_{j i, j}-\widehat{p}_{i}\right)+\widehat{f}_{i}^{(1)}=\rho_{1}^{0} \widehat{u}_{i}, \\
p^{-2}\left(\widehat{s}_{j i, j}+p_{i}\right)+\widehat{f}_{i}^{(2)}=\rho_{2}^{0} \widehat{w}_{i}, \\
p^{-2}\left[2 \widehat{m}_{j i, j}+\varepsilon_{i j k}\left(\widehat{t}_{j k}+\widehat{s}_{j k}\right)-2 \widehat{h}_{i}\right]+2 \widehat{g}_{i}^{(1)}=2 J_{i j}^{(2)} \widehat{\varphi}_{j}, \\
p^{-2}\left[2 \widehat{\mu}_{j i, j}+\varepsilon_{i j k}\left(\widehat{t}_{j k}+\widehat{s}_{j k}\right)+2 \widehat{h}_{i}\right]+2 \widehat{g}_{i}^{(2)}=2 J_{i j}^{(2)} \widehat{\psi}_{j} .
\end{gathered}
$$

In view of (3), (4), (34), and (38), we have

$$
\begin{aligned}
& A_{i j m n}\left(\widehat{\varepsilon}_{m n}^{\prime} \widehat{\varepsilon}_{i j}+\widehat{\varepsilon}_{m n} \widehat{\varepsilon}_{i j}^{\prime}\right)+2 B_{i j m n}\left(\widehat{\kappa}_{m n} \widehat{\varepsilon}_{i j}^{\prime}+\widehat{\kappa}_{m n}^{\prime} \widehat{\varepsilon}_{i j}\right) \\
& +C_{i j m n}\left(\widehat{\kappa}_{m n} \widehat{\kappa}_{i j}^{\prime}+\widehat{\kappa}_{m n}^{\prime} \widehat{\kappa}_{i j}\right)+D_{i j m n}\left(\widehat{e}_{m n} \widehat{e}_{i j}^{\prime}+\widehat{e}_{m n}^{\prime} \widehat{e}_{i j}\right) \\
& +2 E_{i j m n}\left(\widehat{\gamma}_{m n} \hat{e}_{i j}^{\prime}+\widehat{\gamma}_{m n}^{\prime} \widehat{e}_{i j}\right)+F_{i j m n}\left(\widehat{\gamma}_{m n} \widehat{\gamma}_{i j}^{\prime}+\widehat{\gamma}_{m n}^{\prime} \widehat{\gamma}_{i j}\right) \\
& +2 G_{i j m n}\left(\widehat{e}_{m n} \widehat{\varepsilon}_{i j}^{\prime}+\widehat{e}_{m n}^{\prime} \widehat{\varepsilon}_{i j}\right)+2 H_{i j m n}\left(\widehat{\gamma}_{m n} \widehat{\varepsilon}_{i j}^{\prime}+\widehat{\gamma}_{m n}^{\prime} \widehat{\varepsilon}_{i j}\right) \\
& +2 J_{i j m n}\left(\widehat{\kappa}_{m n} \widehat{\varepsilon}_{i j}^{\prime}+\widehat{\kappa}_{m n}^{\prime} \widehat{\varepsilon}_{i j}\right)+2 L_{i j m n}\left(\widehat{\gamma}_{m n} \widehat{\varepsilon}_{i j}^{\prime}+\widehat{\gamma}_{m n}^{\prime} \widehat{\varepsilon}_{i j}\right) \\
& +2 a_{i j m}\left(\widehat{d}_{m} \widehat{\varepsilon}_{i j}^{\prime}+\widehat{d}_{m}^{\prime} \widehat{\varepsilon}_{i j}\right)+2 b_{i j m}\left(\widehat{d}_{m} \widehat{e}_{i j}^{\prime}+\widehat{d}_{m}^{\prime} \widehat{e}_{i j}\right) \\
& +2 c_{i j m}\left(\widehat{d}_{m} \widehat{\kappa}_{i j}^{\prime}+\widehat{d}_{m}^{\prime} \widehat{\kappa}_{i j}\right)+2 f_{i j m}\left(\widehat{d}_{m} \widehat{\gamma}_{i j}^{\prime}+\widehat{d}_{m}^{\prime} \widehat{\gamma}_{i j}\right) \\
& +2 g_{i j m}\left(\widehat{\xi}_{m} \widehat{\varepsilon}_{i j}^{\prime}+\widehat{\xi}_{m}^{\prime} \widehat{\varepsilon}_{i j}\right)+2 h_{i j m}\left(\widehat{\xi}_{m} \widehat{e}_{i j}^{\prime}+\widehat{\xi}_{m}^{\prime} \widehat{e}_{i j}\right) \\
& +2 \ell_{i j m}\left(\widehat{\xi}_{m} \widehat{\kappa}_{i j}^{\prime}+\widehat{\xi}_{m}^{\prime} \widehat{\kappa}_{i j}\right)+2 n_{i j m}\left(\widehat{\xi}_{m} \widehat{\gamma}_{i j}^{\prime}+\widehat{\xi}_{m}^{\prime} \widehat{\gamma}_{i j}\right) \\
& +a_{i j}\left(\widehat{d}_{j} \widehat{d}_{i}^{\prime}+\widehat{d}_{j}^{\prime} \widehat{d}_{i}\right)+b_{i j}\left(\widehat{\xi}_{j} \widehat{\xi}_{i}^{\prime}+\widehat{\xi}_{j}^{\prime} \widehat{\xi}_{i}\right)+2 c_{i j}\left(\widehat{\xi}_{j} \widehat{d}_{i}^{\prime}+\widehat{\xi}_{j}^{\prime} \widehat{d}_{i}\right) \\
& =2\left(\widehat{t}_{i j} \widehat{\varepsilon}_{i j}^{\prime}+\widehat{s}_{i j} \hat{e}_{i j}^{\prime}+\widehat{m}_{i j} \widehat{\kappa}_{i j}^{\prime}+\widehat{\mu}_{i j} \widehat{\gamma}_{i j}^{\prime}+\widehat{p}_{i} \widehat{d}_{i}^{\prime}+\widehat{h}_{i} \widehat{\xi}_{i}^{\prime}\right) \\
& =2\left[\left(\widehat{t}_{j i} \widehat{u}_{i}^{\prime}+\widehat{s}_{j i} \widehat{w}_{i}^{\prime}+\widehat{m}_{j i} \widehat{\varphi}_{i}^{\prime}+\widehat{\mu}_{j i} \widehat{\psi}_{i}^{\prime}\right)_{, j}\right. \\
& +p^{2} \widehat{f}_{i}^{(1)} \widehat{u}_{i}^{\prime}+p^{2} \widehat{f}_{i}^{(2)} \widehat{w}_{i}^{\prime}+p^{2} \widehat{g}_{i}^{(1)} \widehat{\varphi}_{i}^{\prime} \\
& +p^{2} \widehat{g}_{i}^{(2)} \widehat{\psi}_{i}^{\prime}-\rho_{1}^{0} p^{2} \widehat{u}_{i} \widehat{u}_{i}^{\prime}-\rho_{2}^{0} p^{2} \widehat{w}_{i} \widehat{w}_{i}^{\prime} \\
& \left.-p^{2} J_{i j}^{(1)} \widehat{\varphi}_{j} \widehat{\varphi}_{i}^{\prime}-p^{2} J_{i j}^{(2)} \widehat{\psi}_{j} \widehat{\psi}_{i}^{\prime}\right] .
\end{aligned}
$$

With the aid of the divergence theorem and taking into account that $P$ is a solution of the problem, from (32), (37), (39), and (34) we find that

$$
\begin{aligned}
& \Phi_{g}\left(s^{*}\right)=\Phi_{g}(s) \\
&+\int_{B} \int_{0}^{\infty} p^{-2} G(p) \\
&+\left[A_{i j m n} \widehat{\varepsilon}_{m n}^{\prime} \widehat{\varepsilon}_{i j}^{\prime}+2 B_{i j m n} \widehat{\kappa}_{m n}^{\prime} \widehat{\varepsilon}_{i j}^{\prime}\right. \\
&+C_{i j m n} \widehat{\kappa}_{m n}^{\prime} \widehat{\kappa}_{i j}^{\prime}+D_{i j m n} \widehat{e}_{m n}^{\prime} \widehat{e}_{i j}^{\prime} \\
&+2 E_{i j m n} \widehat{\gamma}_{m n}^{\prime} \widehat{e}_{i j}^{\prime}+F_{i j m n} \widehat{\gamma}_{m n}^{\prime} \widehat{\gamma}_{i j}^{\prime} \\
&+2 G_{i j m n} \widehat{e}_{m n}^{\prime} \widehat{\varepsilon}_{i j}^{\prime}+2 H_{i j m n} \widehat{\gamma}_{m n} \widehat{\varepsilon}_{i j}^{\prime} \\
&+2 J_{i j m n} \widehat{\kappa}_{m n}^{\prime} \widehat{\varepsilon}_{i j}^{\prime}+2 L_{i j m n} \widehat{\gamma}_{m n}^{\prime} \widehat{\varepsilon}_{i j}^{\prime}
\end{aligned}
$$




$$
\begin{aligned}
& +2 a_{i j m} \widehat{d}_{m}^{\prime} \widehat{\varepsilon}_{i j}^{\prime}+2 b_{i j m} \widehat{d}_{m}^{\prime} \widehat{e}_{i j}^{\prime} \\
& +2 c_{i j m} \widehat{d}_{m}^{\prime} \widehat{\kappa}_{i j}^{\prime}+2 f_{i j m} \widehat{d}_{m}^{\prime} \widehat{\gamma}_{i j}^{\prime} \\
& +2 g_{i j m} \widehat{\xi}_{m}^{\prime} \widehat{\varepsilon}_{i j}^{\prime}+2 h_{i j m} \widehat{\xi}_{m}^{\prime} \widehat{e}_{i j}^{\prime} \\
& +2 \ell_{i j m} \widehat{\xi}_{m}^{\prime} \widehat{\kappa}_{i j}^{\prime}+2 n_{i j m} \widehat{\xi}_{m}^{\prime} \widehat{\gamma}_{i j}^{\prime} \\
& +a_{i j} \widehat{d}_{j}^{\prime} \widehat{d}_{i}^{\prime}+b_{i j} \widehat{\xi}_{j}^{\prime} \widehat{\xi}_{i}^{\prime}+2 c_{i j} \widehat{\xi}_{j}^{\prime} \widehat{d}_{i}^{\prime} \\
& +p^{2}\left(\rho_{1}^{0} \widehat{u}_{i}^{\prime} \widehat{u}_{i}^{\prime}+\rho_{2}^{0} \widehat{w}_{i}^{\prime} \widehat{w}_{i}^{\prime}+J_{i j}^{(1)} \widehat{\varphi}_{j}^{\prime} \widehat{\varphi}_{i}^{\prime}\right. \\
& \left.\left.+J_{i j}^{(2)} \widehat{\psi}_{j}^{\prime} \widehat{\psi}_{i}^{\prime}\right)\right] d p d v_{x} .
\end{aligned}
$$

From (40) and hypotheses $\left(A_{2}\right)$ and (v) we conclude that (33) holds.

In a similar way we can establish a minimum principle for the problem characterized by the following boundary conditions:

$$
\begin{aligned}
u_{i} & =\widetilde{u}_{i}, \quad w_{i}=\widetilde{w}_{i} \quad \text { on } \bar{S}_{1} \times I, \\
t_{j i} n_{j} & =\tilde{t}_{i}, \quad s_{j i} n_{j}=\widetilde{s}_{i} \quad \text { on } S_{2} \times I, \\
\varphi_{i} & =\widetilde{\varphi}_{i}, \quad \psi_{i}=\widetilde{\psi}_{i} \quad \text { on } \bar{S}_{3} \times I, \\
m_{j i} n_{j} & =\widetilde{m}_{i}, \quad \mu_{j i} n_{j}=\widetilde{\mu}_{i} \quad \text { on } S_{4} \times I,
\end{aligned}
$$

where $\widetilde{u}_{i}, \widetilde{w}_{i}, \widetilde{\varphi}_{i}, \widetilde{\psi}_{i}, \widetilde{t}_{i}, \widetilde{s}_{i}, \widetilde{m}_{i}$, and $\tilde{\mu}_{i}$ are prescribed functions.

\section{Conclusions}

In the present work, a theory of composites modelled as mixtures of two elastic Cosserat continua is investigated. A counterpart of the principle of minimum potential energy of the classical elastostatics is presented. In the dynamic theory, the solution of the boundary-initial-value problem is characterized by equations which incorporate the initial conditions. A minimum principle for the solution of dynamic problems is established.

\section{Conflict of Interests}

The authors declare that they have no competing interests.

\section{Authors' Contribution}

The authors equally contributed to this work.

\section{Acknowledgment}

The authors express their gratitude to the referees for their helpful suggestions.

\section{References}

[1] S. J. Allen and K. A. Kline, "A theory of mixtures with microstructure," Zeitschrift für Angewandte Mathematik und Physik, vol. 20, no. 2, pp. 145-155, 1969.

[2] R. J. Twiss and A. C. Eringen, "Theory of mixtures for micromorphic materials-I. Balance laws," International Journal of Engineering Science, vol. 9, no. 10, pp. 1019-1044, 1971.

[3] R. J. Twiss and A. C. Eringen, "Theory of mixtures for micromorphic materials-II. Elastic constitutive equations," International Journal of Engineering Science, vol. 10, no. 5, pp. 437-465, 1972.

[4] N. T. Dunwoody, "Balance laws for liquid crystal mixtures," Zeitschrift für Angewandte Mathematik und Physik ZAMP, vol. 26, no. 1, pp. 105-117, 1975.

[5] A. Bedford and D. S. Drumheller, "Theories of immiscible and structured mixtures," International Journal of Engineering Science, vol. 21, no. 8, pp. 863-960, 1983.

[6] A. C. Eringen, "Micropolar mixture theory of porous media," Journal of Applied Physics, vol. 94, no. 6, pp. 4184-4190, 2003.

[7] M. F. McCarthy and H. F. Tiersten, "A theory of viscoelastic composites modeled as interpenetrating solid continua with memory," Archive for Rational Mechanics and Analysis, vol. 81, no. 1, pp. 21-51, 1983.

[8] R. N. Hills and P. H. Roberts, "On the formulation of diffusive mixture theories for two-phase regions," Journal of Engineering Mathematics, vol. 22, no. 2, pp. 93-106, 1988.

[9] J. Aboudi, "Micromechanical modeling of finite viscoelastic multiphase composites," Zeitschrift für angewandte Mathematik und Physik ZAMP, vol. 51, no. 1, pp. 114-134, 2000.

[10] D. Ieşan, "On the theory of viscoelastic mixtures," Journal of Thermal Stresses, vol. 27, no. 12, pp. 1125-1148, 2004.

[11] A. Bedford and D. S. Drumheller, "A variational theory of immiscible mixtures," Archive for Rational Mechanics and Analysis, vol. 68, no. 1, pp. 37-51, 1978.

[12] H. Gouin, "Variational theory of mixtures in continuum mechanics," European Journal of Mechanics. B Fluids, vol. 9, no. 5, pp. 469-491, 1990.

[13] D. Ieşan and R. Quintanilla, "On a theory of interacting continua with memory," Journal of Thermal Stresses, vol. 25, no. 12 , pp. 1161-1177, 2002.

[14] S. De Cicco and G. Iaccarino, "Minimum principle for mixtures with memory," Mechanics Research Communications, vol. 34, no. 4, pp. 331-337, 2007.

[15] L. Placidi, F. Dell'Isola, N. Ianiro, and G. Sciarra, "Variational formulation of pre-stressed solid-fluid mixture theory, with an application to wave phenomena," European Journal of Mechanics. A. Solids, vol. 27, no. 4, pp. 582-606, 2008.

[16] M. E. Gurtin, “The linear theory of elasticity," in Handbuch der Physik, C. Truesdell, Ed., vol. VIa/2, pp. 1-295, Springer, Berlin, Germany, 1972.

[17] R. Reiss, "Minimum principles for linear elastodynamics," Journal of Elasticity, vol. 8, no. 1, pp. 35-46, 1978.

[18] R. Reiss and E. J. Haug, "Extremum principles for linear initialvalue problems of mathematical physics," International Journal of Engineering Science, vol. 16, no. 4, pp. 231-251, 1978. 


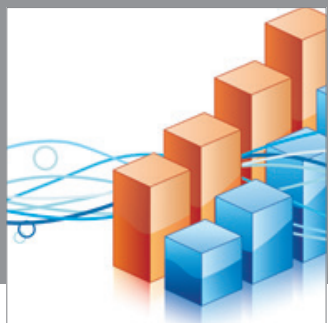

Advances in

Operations Research

mansans

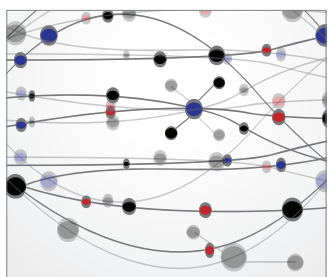

The Scientific World Journal
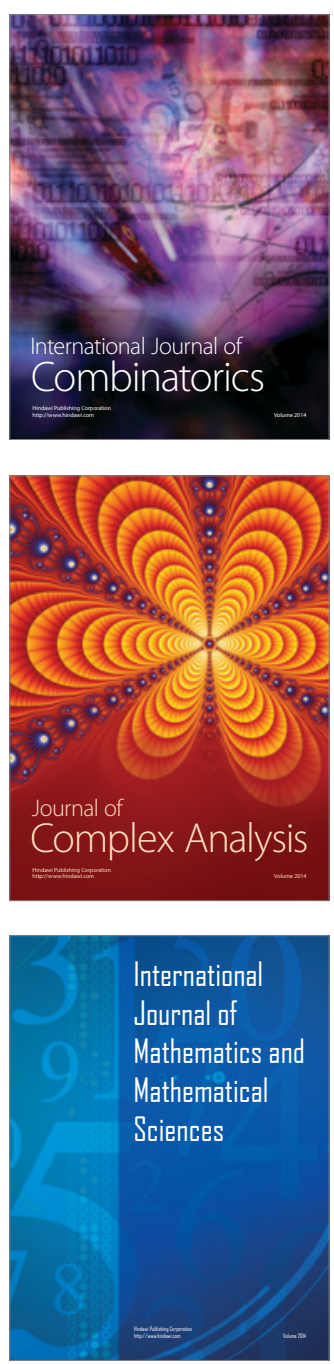
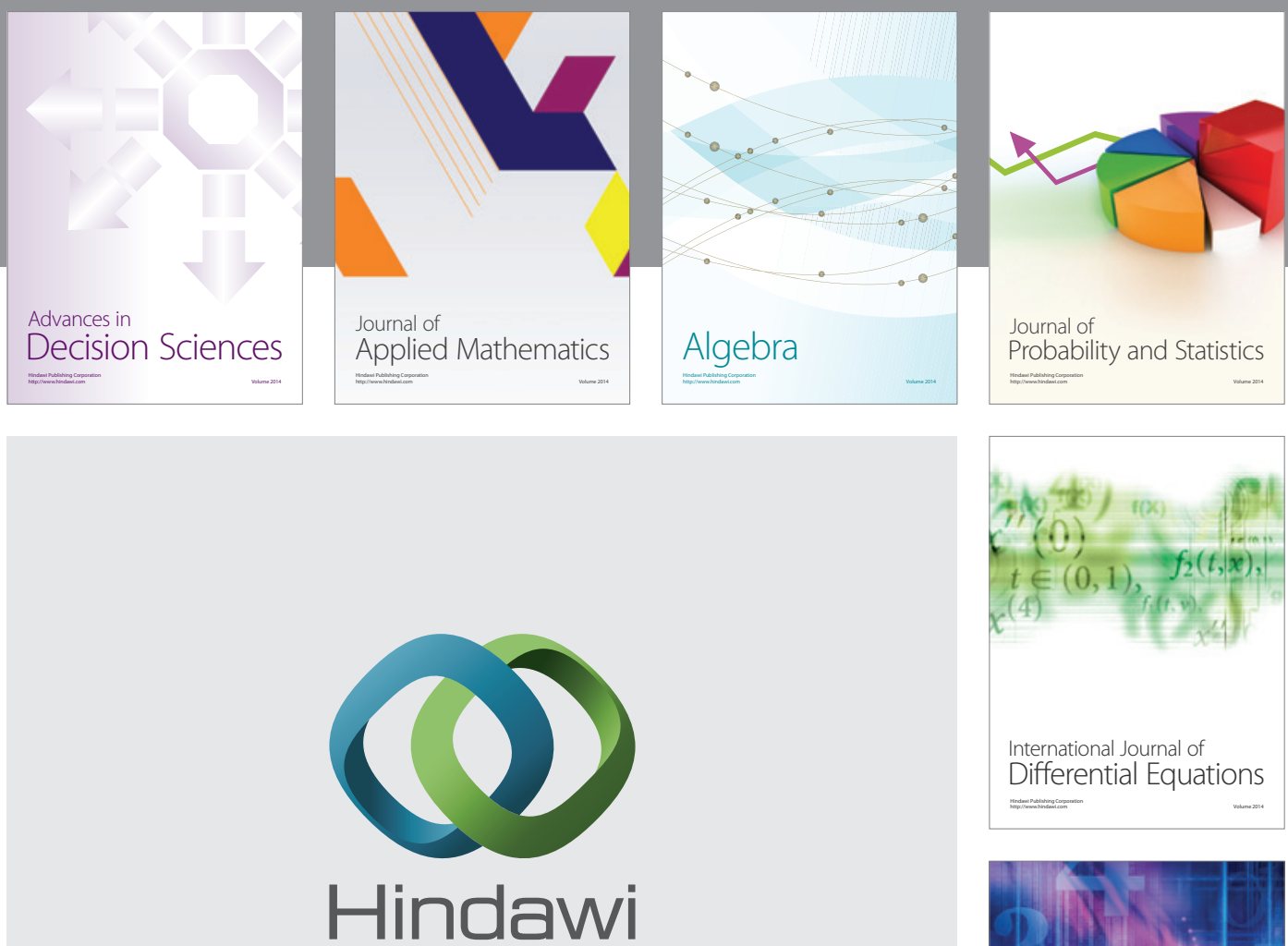

Submit your manuscripts at http://www.hindawi.com
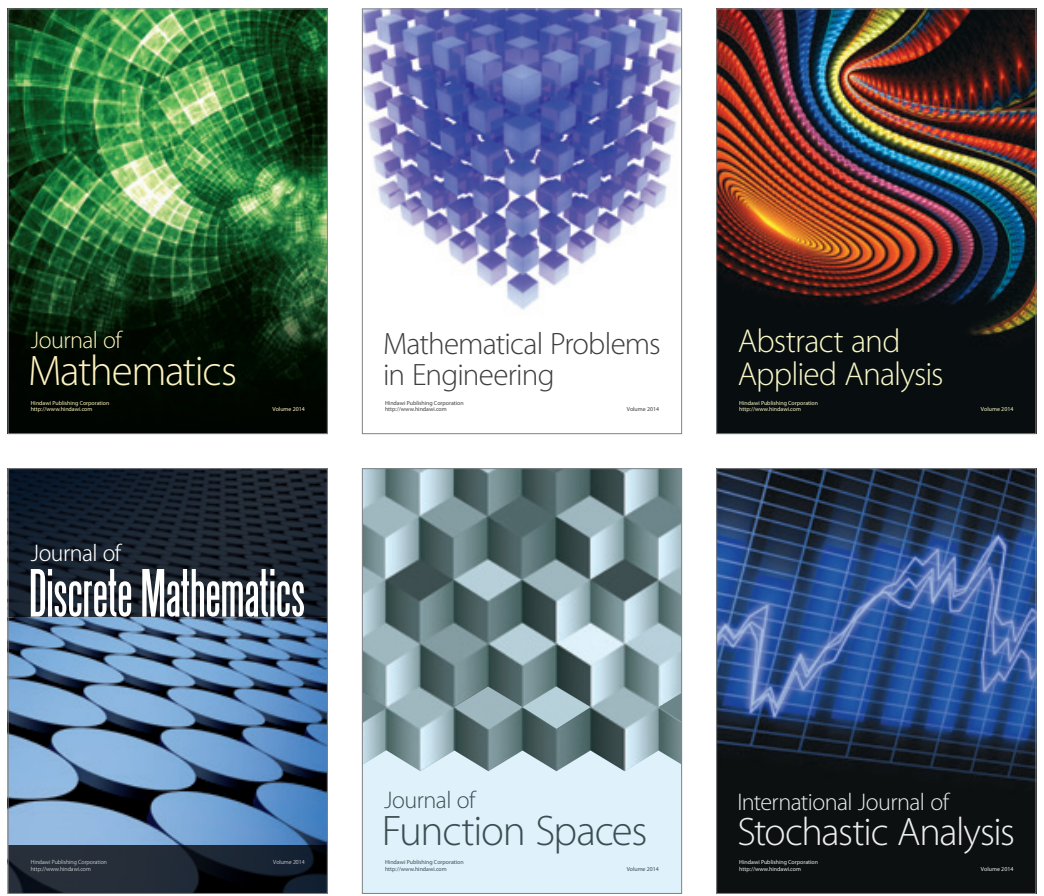

Journal of

Function Spaces

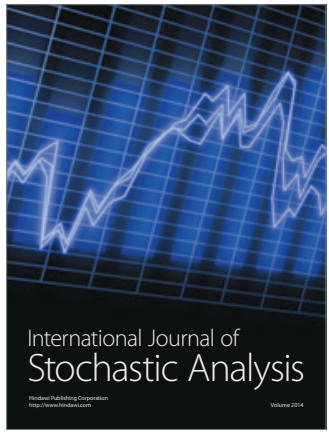

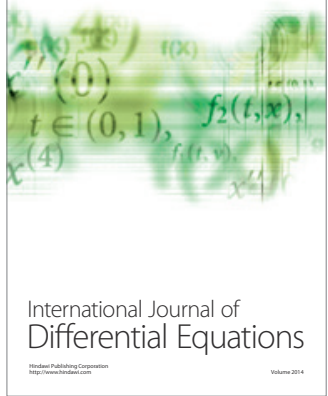
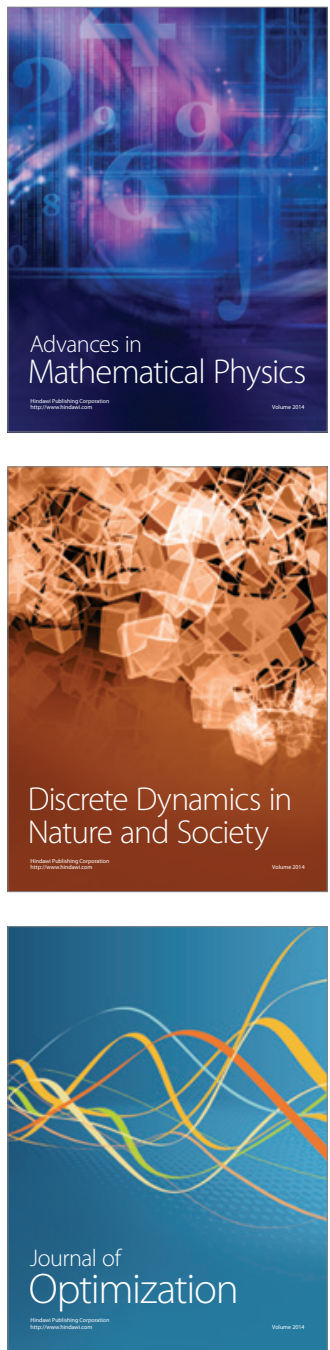